\title{
ANAEROBIC STAGE OF THE EVOLUTIONARY DEVELOPMENT OF ANIMAL CELLS
}

\author{
Alexander R. Zaritsky, Marina N. Kirichenko \\ Lebedev Physics Institute, Russian Academy of Sciences, http://www.lebedev.ru \\ 119991 Moscow, Russian Federation \\ zaritsky@sci.lebedev.ru
}

Vladimir I. Grachev

Kotel'nikov Institute of Radio Engineering and Electronics, Russian Academy of Sciences, http://www.cplire.ru 11/7, Mokhovaya str., 125009 Moscow, Russian Federation

grachev@cplire.ru

\section{Yuri P. Vorontsov}

Filatov Hospital no. 13, Moscow Department of Healthcare, http://www.13dgkb.ru

103001 Moscow, Russian Federation

yupmkpsp@rambler.ru

\section{Vyacheslav S. Pronin}

Sechenov First Moscow State Medical University, Ministry of Healthcare and Social Development of RF,

http://www.mmm.ru

119991 Moscow, Russian Federation

vspronin@yandex.ru

Abstract. An analysis of the likely evolution of living world in the period of formation of the first animal cells is presented. Conditions and possible way of transformation of lipid vesicles into nucleated cells of animals are shown. The main content of the evolutionary path at the considered stage is postulated to be formation of the molecular mechanisms of energy metabolism in two modes: mode of activity and division (mitosis) of the vesicles. We identify leading physicalchemical factors governing order of changes of processes and phenomena during cell division acidity of the cytoplasm and concentration of the main macroerg - ATP. On the phase diagrams of the kinetics of energy metabolism of lipid vesicles and first cells cyclic processes of modes of activation and mitosis are illustrated under conditions of continuous synthesis and enzymatic breakdown of glycogen in the cytoplasm, regulatory inhibitors and activators of metabolic processes, the concentration of ATP and hydrogen ions, involved in running of homeostasis. Selforganizing nature of the kinetics of energy metabolism of lipid vesicles cytoplasm and formed first archaic cells are shown

Keywords: anaerobic stage of evolution, lipid vesicles, viruses, modes of energy metabolism, acidity, ATP, phase diagrams of metabolism

PACS: 87.23. Kg

Bibliography - 6 references

Received 28.04.2015

RENSIT, 2015, 7(1):87-99

DOI: $10.17725 /$ rensit.2015.07.087

\section{CONTENTS}

1. IntRoduction (88)

2. Conditions of evolutionary development OF CELLS BEFORE ATMOSPHERE OXYGEN ACCUMULATION (88)

3. AdJUSTMENT OF THE ACTIVITY MODE, TRANSITION TO THE CELL DIVISION (89)

4. Mitosis of ARChaic CElls (91)
5. Evolution OF THE KINETICS OF THE MAIN PARAMETERS OF THE ENERGY METABOLISM OF LIPID VESICLES AND FIRS CELLS (95)

6. Self assembling of the kinetics of METABOLISM MODE (97)

7. Conclusions (98)

REFERENCES (99) 


\section{INTRODUCTION}

In this paper, an analysis of the problem of probable evolutionary development of animal nucleated cells is represented. The basic principles of this analysis are as follows:

- new information is contributed to the cells by viruses in the process of symbiotic and antagonistic interactions with them;

- new information saved forever, appearing as an evolutionary memory, and can be saved in the cells only in case of the energy gain obtained with the appearance of new structures by saving or intensification (general line of development of living systems) of the free energy generation in the cells, without damage of existing structures;

- preservation of native (the integrity of the composition and properties) organic structures, with a few exceptions, is maintained in a fluid homeostasis in the cells, in a continuous synthesis and enzymatic disassembling of substances.

The objective of the analysis is to ascertain the sources of the appearance, formation and improvement of facilities and structures of the regulatory mechanisms that ensured to archaic cells:

- high reliability in the accumulation of glycogen, sufficient to power the processes of cell division;

- well-timed (when conditions appear) automatic switching of two modes of metabolism that were already developed from the mode of activity to division and back.

Moreover, it is necessary to identify the driving forces and the main factors determining the procedure for changing of processes and events during the mitosis and for switching over two modes of metabolism in the cells.

\section{CONDITIONS OF EVOLUTIONARY DEVELOPMENT OF CELLS BEFORE ATMOSPHERE OXYGEN ACCUMULATION}

Papers [1,2] show separation of archaic cycle of the processes of energy metabolism into cycle of activity and cycle of division in the first nucleated cells. The formation and improvement of metabolism of these two modes apparently started at a partial pressure of atmospheric oxygen, not more than $2 \mathrm{~mm} H g$ (judging by the level of oxygen homeostasis in the cell cytoplasm of modern animal). The overwhelming dominance of glucose in the aquatic environment, surrounding of lipid vesicles, contributed to their evolutionary transformations. At the considered period, glucose, like a power-consuming substrate, appeared to be evolutionarily first and the main product of photosynthetic lipid vesicles - precursors of plant cells and their organelles. At this time cells of fungi and plants have not yet formed, and oxygen in the atmosphere was just starting to accumulate as a result of activity of named vesicles. First cells had not the prerequisites for the formation of the enzyme system of aerobic sugar digestion at that period. Therefore, the energy basis of the mitotic activity of the first animal cells was an anaerobic digestion of glucose and its polymer - glycogen ${ }^{1}$. Glycolysis, accompanying by the synthesis of ATP, had been formed and preserved as a result of formation and improvement of the respective enzyme system of anaerobic glucose digestion in the mitotic cycle of lipid vesicles. Here, the biological conservatism gives full play.

Improvement of the energy metabolism ${ }^{2}$ of lipid vesicles was determined by the appearance of the regulation mechanisms of its modes. By the time, two modes of metabolism of cyclic

\footnotetext{
${ }^{1}$ Stay the same up to date.

${ }^{2}$ Energy metabolism is considered as two groups of energy dependent and oppositely directed processes: therelease of free energy and its accumulation in macroergs with further consumption of this energy for cell survival and its adaptation to environmental niche.
} 
nature formed - the mode of activity and mitosis. Transitions from one mode to another and back had the random and probabilistic character. Often, protocells started their division without sufficient energy supply (glycogen) and died. On the other hand, the delay in the mode of activity could lead to unlimited glycogen accumulation. This could significantly change conditions of the occurrence of metabolic processes up to their discrimination and death of protocells.

To control these transitions, an appropriate mechanism had been developed. The foundation for this mechanism was an interspecies struggle of virus with cells for energy reserves. In the process of the development of living world there was lack of energy for sustainable reproduction of increasingly large viruses because energy accumulated in the cycles of activity of protocells during the time limited by unregulated transfers it to division. It was the large viruses that brought in cells information about regulatory substances - inhibitors and activators (peptide nature) of the special group of enzymes alkalizing protocell cytoplasm. Nowadays it is this group of enzymes that catalyzes reactions of high molecular organic compounds synthesis with absorption of $\mathrm{H}^{+}$ ions [2], leading to alkalinization of the reaction medium. We recall that at the considered evolutionary period the appearance and the action of such enzymatic reactions in the cytoplasm allowed the first cells to intensify the processes of energy metabolism and to separate in time two major groups of processes competing for using of basic macroergs [1]. Such separation made possible realization of cyclical modes in the cells. The influence of the activators and inhibitors on the rate of alkalizing of the cytoplasm in the cells ensures the formation and operation of appropriate regulatory mechanisms, indirectly, through changes in the acidity of the environment, affecting the speed and volume of glycogen stored.

\section{ADJUSTMENT OF THE ACTIVITY MODE, TRANSITION TO THE CELL DIVISION}

Let to consider the possible scheme of action of the regulatory inhibitors and activators. Functional role of the activity mode in the archaic cells consisted in the glycogen accumulation. Therefore, part of the generated power was given to them for this purpose. The rest of energy is spent for maintaining of the flowing homeostasis. All the compounds (except for $D N A$ ) were continuously synthesized in a protocell and simultaneously subjected to enzymatic degradation. Equality of the rates of these processes average over the cycle of activity provided a constant amount and concentration of the compounds involved in the homeostasis maintaining. Such a flow of matter, but with a feature, was set for regulatory inhibitors and activators. Partial inhibition of alkalizing process by new regulatory substances made spontaneous switching of metabolism from the cycle of activity to mitosis possible at the phase of its ATP concentration growing from the beginning of accumulation of the glycogen. Activators of alkalizing process were necessary to fine-tune of the depth of the initial inhibition. In the process of natural selection of the inhibitors only those compounds which have affinity for glycogen were chosen. This permitted to distract part of the inhibitors synthesized in the cell for the formation of inhibitor-glycogen complexes during all the time of glycogen synthesis and accumulation. As the increasing of the amount of glycogen "bushes" in the cell, the rate of appearance of the new sites of sorption for these inhibitors continuously increased. This provided a reduction in their concentration in the cytoplasm, and as a consequence, an increasing of the activity of enzymes involved in the process of alkalization. Therefore, the speed of the alkalizing processes increased, gradually approaching to the constant level that is determined by the concentration of 
the enzymes and their activators. As reaching the neutral $p H$ of cytoplasm, processes of cell division switched on automatically (the mechanisms of switching considered below). In the process of evolutionary improvement of the mechanism, the above-mentioned compounds were selected and preserved until the present day as a part of the homeostasis. They ensured reliable switching of the cell metabolism from the mode of activity with the accumulation of glycogen into the mode of division with its consumption.

Fig. 1 shows changing of ATP concentration and cytoplasm $p H$ values (phase portrait of energy metabolism) at the mode of glycogen accumulation in the archaic animal cell. There is the trajectory of these values changing which determined (determines and nowadays) the rates of metabolic processes in the cytoplasm on the phase portrait $\left\{n_{\text {ATP }} ; p H\right\}$. Starting at the point $A$ with coordinates $n_{\text {int }}$ and $p H_{\text {int }}$ below a critical level $p H_{\mathrm{cr}}$, the state of acidic cytoplasm changed cyclically. On moving along the quasiclosed path, the phase point $\left(n_{\text {ATP }} ; p H\right)$ gradually shifted towards higher $p H$ values. The figure shows three open cycles of activity, which are numbered in the order of phase point passing them. The arrows indicate the direction of

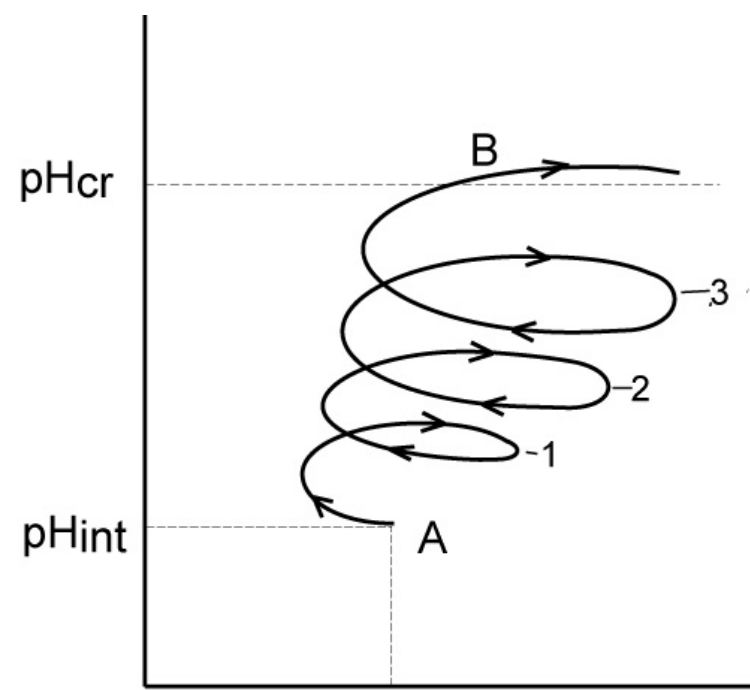

nint

nATP

Fig. 1. Energy metabolism phase portrait of archaic cell in the mode of activity. its movement. If one enter a time axis $t$, the phase point in the space $\left\{n_{\text {ATP }} ; p H ; t\right)$ moving along the helix unwinding, will shift towards higher values $p H$. Upon reaching the critical level - value $p H_{c r}$ at point $B$, mitosis switched automatically.

It should be noted (see Fig. 1) that changing of $n_{\text {ATP }}$ and $p H$ values had oscillatory nature against a monotonic growth of their average values over the cycle as the glycogen accumulation because of the gradual release of inhibition of the processes of cytoplasm alkalizing.

Note also that it is extremely important for our consideration a case of sudden switching of strong oxidation processes which can significantly acidify the cytoplasm, despite of its increasing alkalization. Changing of ATP and the $p H$ values will inevitably similar the way presented in Fig. 1, only in reverse, and at much higher concentrations of ATP.

We cannot ignore the issue of intensification of energy metabolism as a general line of evolutionary transformation of the cells at the stage of its development with regard to the consideration of the activity cycle. Clearly, the maximum of power generation of free energy per cycle can be achieved by alkalizing of the cytoplasm to the level close to neutral $p H$ values, but excluding the transition to the mode of cell division and providing accumulation of glycogen sufficient for mitosis. Therefore, by further improvement of the activity cycle of archaic and modern cells, an extreme (but below the neutral value) position of "alkaline" branch of the phase trajectory of the states on the coordinate plane $\left\{n_{\text {ATP }} ; p H\right\}$ was fixed. Moreover, intensification of energy metabolism of cells in the mode of activity also appeared in the achievement of high values of maximum concentrations of ATP in the cytoplasm and displacement of "acidic" branch of the phase trajectory towards lower $\mathrm{pH}$ values. 


\section{MITOSIS OF ARCHAIC CELLS}

Upon reaching the neutral cytoplasmic $p H$ values, conditions of very early (vesicular) stages of development of the living world were realized in cells. Most of the compounds and their complexes with a high affinity for lipids associated with membrane. It concerned both cytomembranes and membranes of cell nucleus. A little earlier, information molecule underwent a conformational change, taking the form of "lamp brush" convenient for their replication (copying) in the case of slightly acidic cytoplasm. Polynucleotides had not affinity for lipids and could associate with membranes through protein complexes, having the affinity for lipids at such $p \mathrm{H}$ values. Association of these complexes and other compounds with the membranes strengthened enzymatic breakdown of lipids. This breakdown supplied energy for synthesis of macroergs required for DNA replication of the genome. First of all, lipids of the nucleus were subjected to decay up to its disappearance due to the acting of enzymes in two ways: inside and outside ofthe nuclear membrane. When neutral values of environment were achieved, the chromosomes (after replication) condensed, marking the beginning of mitosis, called prophase.

The most important moment in the development of further events was in the sharp increasing of activity of the enzymes, catalyzing reaction of energy metabolism. In accordance with second part of the introduction, enzymes inherited properties of the compounds characteristic for them at the early stages of the evolution of living systems. Since metabolism at that time carried out mainly on the surface of the lipids or in the aqueous medium directly adjacent to them [4], therefore, the evolutionary selection of enzymes was conducted, respectively, at the maximum of their activity in association with lipid layers.

The increase of enzyme activity during their sorption on the surface of the membrane caused an increase in the power of oxidation processes (recall that it was the anaerobic digestion of glucose). The associated increasing of the subsequent acidification of the cytoplasm proved able to overcome the action of the special chain of reactions of its alkalizing. At this evolutionary period, the volume of enzymatic pool of oxidative processes was sufficient to provide a monotonic acidification of the cytoplasm. It should be noted that steady decrease of the $p H$ value superimposed with oscillations typical for the activity cycle, but with a gradual decrease of the amplitude until their complete disappearance. Such fluctuations in the acidity of the cytoplasm on the background of steady acidification in modern animal cells are characteristic for prometaphase mitosis. They are caused by alternating dominance of opposing processes: synthesis of structures of the spindle and enzymatic breakdown of their material. As a result of such changes, microtubules and other structures forming the spindle change their linear dimensions, providing themselves with complex movements (congression [5]). Upon the formation of the spindle on the background of a steady decrease in the $p H$ of the cytoplasm, oscillations permit to operate of the mechanism of search and seizure. Microtubules "found their own" chromosome, having attached their free ends to its centromere and "shaking stranger", if such one is captured. After damping of the $p H$ value oscillations, the synthetic processes began to dominate due to further acidification of the cytoplasm, caused by the enhanced utilization of glycogen stored. The structure of the spindle lengthened, and all the chromosomes moved toward the equator halfway between the poles, leading to their congregation in the plane of the equator. In this case the cell is believed to enter into metaphase of the mitosis [5].

According to our ideas, algorithm (sequence) of switching of the processes in the mitosis phase of the nucleated animal cells formed at the exactly reporting period of the evolution 
of the anaerobic cells. The leading factor in the spontaneous development of complex picture of events in the mitosis was a steadily decreasing in the $p H$ value of the cytoplasm due to increasing of the oxidative processes because of the acceleration of cell glycogen utilization. Increased oxidative processes caused a significant increase in the concentration of $A T P$, so that the phase trajectory of mitosis did not cross the path of the mode of glycogen accumulation. Therefore, at such high concentrations of this macroerg the processes of its consumption dominated.

Cell have a steadily intensification of oxidative processes in the mode of activity, inevitably accompanied by the increasing acidification of the cytoplasm, due to viruses. New information was implanted into the cells by viruses, and it was saved (according to the second part of the introduction) only if it gave an energy gain. The inevitable consequence of the growth of the information volume in the genome was the intensification of energy metabolism and the increasing of acidification of the cytoplasm. Another important consequence was the growth of the rate and reducing of the time of replication of the genetic material units. The procedure for replication seemed strongly associated with a decrease in the $p H$ of the cytoplasm due to the ever-growing level of free-energy arisen as a result of new information entering into cell. Evolutionary development determined the appearance of more and more complex forms of cellular structures and compounds. Accordingly, genetic information is complicated, requiring for its replication large energy input. Therefore, the nuclear membrane of the cells of higher animals and human became a double. Stock of lipids therein for their disposal as the energy-intensive material was enough to power the processes of genome replication and to transport all participants of multicomponent reactions in the reaction areas.
It should be paying attention once again to one important fact. On vesicular (before cell) stage of development of living world viruses in an aqueous medium "recognize" a lipid-free source of energy by acidification of the environment (although weak). Under the influence of this factor viruses dropped their protective protein shell, replicated their information molecules and synthesized the proteins of their safety part. Diffusion of molecules from the site of synthesis (from a source of free energy) to the aqueous medium with neutral $p H$ ensures the recovery of the affinity of the viral structures to each other. The integrity of viruses was repaired by condensation (aggregation) of their parts in an aqueous medium with neutral acidity. Due to the evolutionary memory, known as a biological conservatism (second position of the introduction), the condensation of chromosomes in the cell inevitably began (begins and nowadays) after reaching neutral $p H$ values of the cytoplasm after the replication of the genetic material in the transition of cells from the activity mode to division.

General line of the cell development was a steadily increasing of power generation capacity and hence oxidative processes. Accordingly, the cytoplasm is also increasingly acidified. The activity of enzymes catalyzing reactions of glycogen synthesis grew with decreasing of $p H$ value, making it difficult to achieve neutral $p H$ values in the cycle of activity and to switch to the cycle of division. Neutral $p H$ values of cytoplasm still were achieved in cells via activation of alkalizing processes of the medium by mentioned above activators - the compound having the ability to form complexes with enzymes (to catalyze the reaction of this process), increasing their activity. Only those compounds whose activity was increased with growing of the plasma $p H$ values were chosen as a result of natural selection. As approaching of the $p H$ values of medium to neutral ones, efficiency of activation of the alkalizing processes 
continuously increased, providing a reliable transition of cells to division. However, such an increasing of medium $p H$ at the beginning of the glycogen accumulation was a significant obstacle to this process. The problem has been removed when the above-mentioned inhibitors of the process of cytoplasm alkalizing were introduced and fixed in the cells. Their activity decreased with increasing of $p H$ (as decreases and nowadays), providing optimum conditions for the synthesis glycogen.

Changing of $p H$ has become the main regulatory factor, determining the metabolism modes changing and procedure for switching of processes in the complex phenomenon of cell division. From these positions, we can give simple explanation for virtually all known facts in the development of events during mitosis in modern cells. After reaching of the limit of cytoplasm alkalizing powerful oxidizing processes switch on due to association of the majority of enzymes of energy metabolism with lipid membrane. As a result, steadily acidifying imposes strict order of transition from one phase of mitosis to another. The mechanism is based on flow of substance, energy and information. For example, as decreasing of cytoplasm $p H$ value the process of assembly of structures initially dominates, gradually slowing down. After that as growing of cytoplasm $p H$ value, enzymatic breakdown takes place, gradually accelerating. Such structures can appear for some time and then disappear. The most mysterious phenomena that have not adequate coverage in the scientific literature from the position of the driving forces are caryokinesis (gr. kario kernel; kinesis - division) and cytokinesis.

Let's start with cytokines. At the considered evolutionary period first cells had not cytoskeleton. Influx (synthesis) of the membrane lipid carried in the inner layer, and its drain (enzymatic breakdown of lipids and outflow of lipids part in aqueous medium) - from their outer and inner layers. Therefore, the density of dipoles heads of lipids in the inner layer was always higher than in the outer layer. Due to this fact the elastic energy and the energy of the electrostatic field appeared in the membrane. Elastic energy tried to reverse the membrane "inside out". Calculations of free energy in accordance with the different contributions of electrostatic and elastic components to it have shown that the minimum of free energy is realized for the form of dumbbell with the constriction in the center. Such constriction could easily be torn in the shear flows in the aquatic environment and two identical cells separated from each other. Note that the variation of the contribution of the electrostatic component to the free energy of the first cell membranes during mitosis inevitably took place automatically due to steady acidification of the cytoplasm. Indeed, as achieving of neutral $p H$ values in the cytoplasm the vast majority of enzymes associated with the membrane. The density of electrostatic energy in lipids was determined by the transmembrane potential difference and the thickness of the membrane (including compounds associated with it). As acidification of the cytoplasm compounds lost their affinity with lipids in the membrane and went into the aquatic environment. The thickness of the membrane is reduced, and the contribution of the electrostatic component to the free energy of the membrane is continuously growing, reaching a critical value. At this moment the cells took the form of dumbbells with the constriction, and two parts of cell separates from each other. Selection of the appropriate affinity of enzymes to the lipid membranes upon different cytoplasmic $p H$ values was also provided by natural selection.

It should be pointed out that another circumstance contributed to the appearance and development of the constriction. Interaction of two groups of enzymes led to accumularion of lipids on the inner side of the membrane at different places. One group of enzymes, 
catalyzing the processes of lipids synthesis, located on the poles of the cell, and another group, catalyzing their decay, - on the equator. Therefore, activity of the first group resulted in an increase of the area of lipids near the poles, and the second - its reduction near the equator. The aqueous medium was extruded towards the poles, contributing to the appearance of the constriction. We state that at an early stage of evolution the first method (changing of the field in membrane) of the constriction forming dominated, and at the subsequent stages, especially after the appearance of the cytoskeleton - the second.

We also cannot ignore the problem of nuclear cell division. In fact, one can observe the process of cell division within another cell. First, we should answer the question: why does nucleus divide first (cell inside the cell), and then the cell itself?

Since oxidation processes occurred in the living cells, these processes were a source of acid products diffusing into water surrounding the cells. Wherein, the middle of the cell, i.e. internal environment of the nucleus, was the most acidic area. Therefore, all events of mitosis began in the dividing nucleus earlier than in the cell since sequence of events is determined by the steadily increasing of the acidity of the internal liquid medium. After nucleus division the cytoplasm acidity continues to increase, and the cell itself is divided. It should be noted that such a sequence of events can only occur if reserves of lipids in the nuclear membrane is sufficient to provide energy for the processes of replication of the genetic material and the rest of them ensures membrane integrity. In addition, after the nucleus division $p H$ value of the internal environment of the cell should satisfy two conditions. First of all, this value should not be below the level providing the beginning of the spindle formation. Secondly, the acidity of the cytoplasm should steadily increase before turning on processes of its alkalizing, allowing completion of all mitotic processes.

Let's return to the mitosis of the first cells, or rather to the end of their division. It has been shown that the reliability of the switching of cell metabolism to the division mode is ensured by activation of the process of cytoplasm alkalizing. As mentioned above the activity of these compounds grew with increasing of $p H$ value of the medium. Exactly such manner provides transition from the mode of activity to the division. Subsequent increasing of the acidity of the internal environment determines the order of the processes changing during mitosis. Beginning of the mitotic activity is indicated by the neutral $p H$ value of the internal environment. To pass all stages of mitosis consistently, acidity of cell cytoplasm should change from neutral $p H$ values (beginning of mitosis) down to lower values (the completion of the dividing process). By what means was it achieved? The range and rate of the $p H$ value changing during acidification depends primarily on the amount and activity of a group of enzymes which catalyze oxidative processes in the cell. An increasing of the pool of such enzymes in these conditions leads to growing of the range and the rate of the $p H$ changing. An increasing of the amount of activators, alkalizing medium, leads to the slowdown of its acidification rate. Besides, the range and the rate of $p H$ changing depended (and depend nowadays) on relation between activity of the compounds of the cytoplasm and the $p H$ value. Steady acidification of the cytoplasm is inevitably accompanied by decreasing of the activity of not only enzymes, catalyzing the oxidative processes, but activators of the alkalization processes. By decreasing of the $p H$ value, faster decline in activity of the activators than of the enzyme guaranteed increasing of the range of $p H$ changing. During natural selection only those conditions were chosen that did not prevent to continue the general line of development of living systems - the intensification of energy 
metabolism. At the considered period of time the metabolism of the cells was on the low level and the quantity of glycogen was not enough to realize one division. Therefore, the intensification mentioned above provided the necessary range of the $p H$ value changing sufficient for mitosis completing, transition to the mode of activity and subsequent glycogen accumulation.

\section{EVOLUTION OF THE KINETICS OF THE MAIN PARAMETERS OF THE ENERGY METABOLISM OF LIPID VESICLES AND FIRS CELLS}

Upon considered period of time, the rate of majority biophysical reaction depended on $p H$ value and concentration of the main macroerg - $n_{\text {ATP }}$ Therefore, to get full picture of the dynamics of any main parameter, one should obtain the rate of its growth $\mathrm{V}^{+}$and its decay $\mathrm{V}^{-}$in the dependence of these two values $-p H$ and $n_{\text {ATP }}$. First of all it is necessary to make it for the concentration of ATP and ion $\mathrm{H}^{+}$. The rates mentioned above for the considered parameters are the function of two parameters: for $n_{\text {ATP }}-V_{\text {ATP }}^{+}\left(n_{\text {ATP }} ; p H\right)$ and $V_{\text {ATP }}^{-}\left(n_{\text {ATP }} ; p H\right)$, for concentration of $\mathrm{H}^{+}-\mathrm{V}^{+}{ }_{\mathrm{H}+}\left(n_{\text {АTP }} ; p H\right)$ and $V_{\mathrm{H}+}\left(n_{\mathrm{ATP}} ; p H\right)$, correspondingly. In the space of the processes rate $\left\{V, n_{\mathrm{AT} \Phi}, p H\right\}$, these rates are the surfaces and each points on them corresponds to the point-trace $\left(n_{\mathrm{AT} \Phi} ; p H\right)$ on the phase plane $\left\{n_{\mathrm{AT} \Phi} ; p H\right\}$; and reverse, the point on the phase plane corresponds to the rates $\mathrm{V}+$ and $\mathrm{V}-\mathrm{-}$ points on the surface of the rates. If the surfaces intersect then the trace of their intersection on the phase plane $\left\{n_{\mathrm{AT} \Phi} ; p H\right\}$ is in general the balance curve of the synthesis and decay rates of the ATP. The same thing we can say about the trace of intersection of the rates of growth $V^{+}{ }_{\mathrm{H}+}\left(n_{\mathrm{AT} \Phi} ; p H\right)$ and the decay $V_{\mathrm{H}_{+}}\left(n_{\mathrm{AT \Phi}} ; p H\right)$ of the concentration of ions $\mathrm{H}^{+}$. The points on the distinct parts of the balance curve can be both stable and unstable for each of said pairs of parameters. We will not provide the thorough analysis of $3 D$-graphs. We restrict our

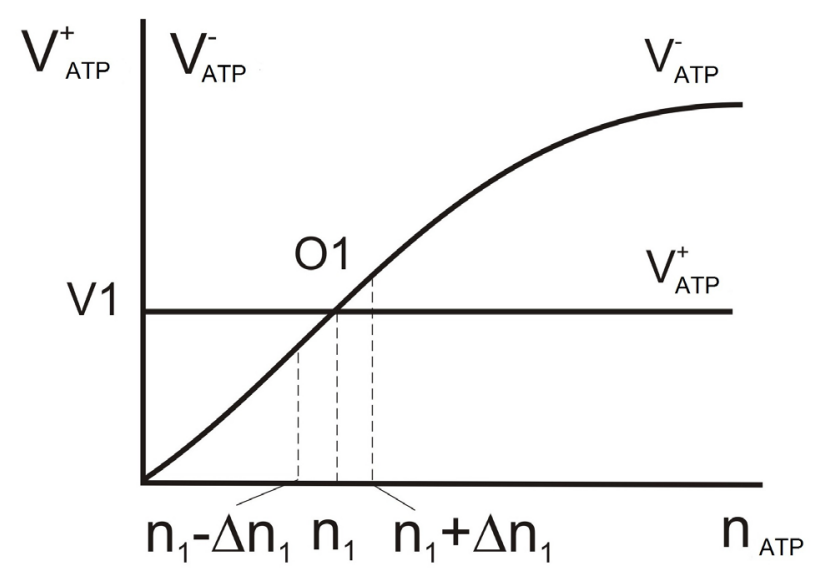

Fig. 2a. Kinetics of energy metabolism of lipid vesicles, stability in $n A T P$.

consideration to the picture of two sections of these graphs in one point on the balance curve.

Let's consider (Fig. 2): first of all, the dependence of $V_{\text {ATP }}^{+}$and $V_{\text {ATP }}^{-}$on concentration of $A T P$ at a constant $p H$ level, and, secondly, dependence of mentioned rates on $p H$ level at the constant ATP concentration. Fig. $2 a$ and Fig. $2 b$ shows the kinetics of energy metabolism of lipid vesicles (they cannot divide) - immediate predecessor of first cells. The first of the figures demonstrates that the point $O_{1}$ with the coordinate $\mathrm{n} 1$ is stable for value of $n_{\text {ATP }}$ and the second one show that this point is unstable for the $p H$ value. Therefore, in the internal medium of the vesicles that possessed such type of metabolism, the small oscillation inevitably excited: for $n_{\text {ATP }}-$ in the range $\left(n_{1}\right.$ - $\left.\Delta n_{1} ; n_{1}+\Delta n_{1}\right)$, where $\Delta n_{1}<<n_{1}$, and for $p H$

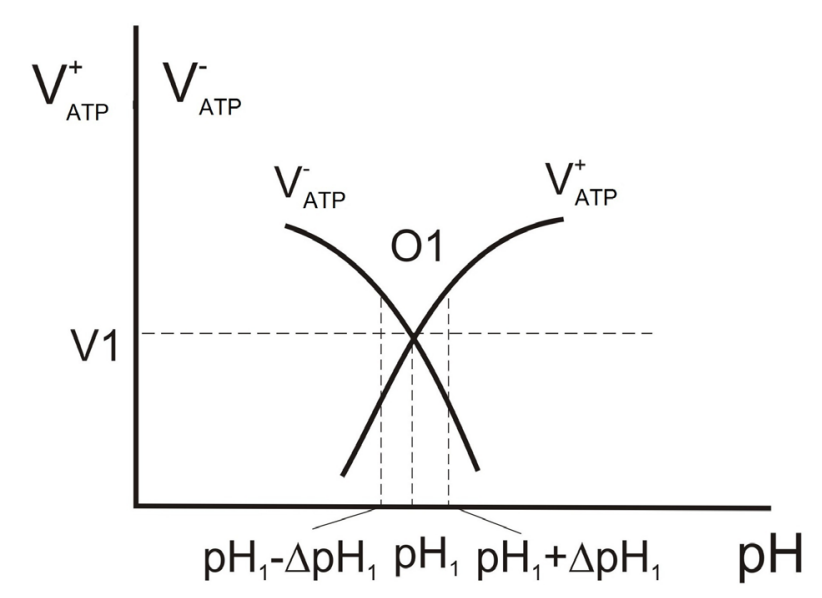

Fig. 2b. Kinetics of the first cell energy metabolism at a constant $p H$ of the cytoplasm. 


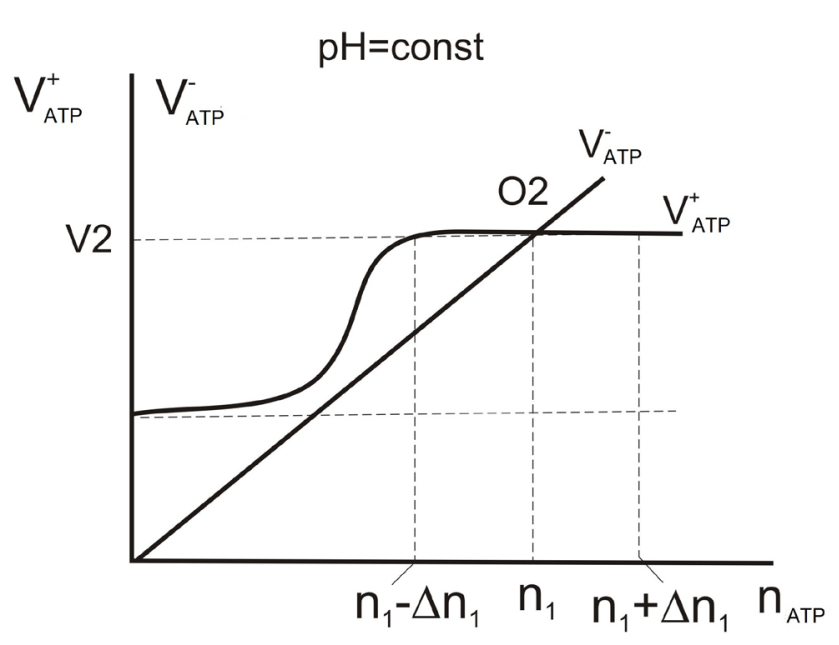

Fig. 2c. Kinetics of energy metabolism of the first cells with a constant of $p H$ of the cytoplasm.

- in the range $\left(p H_{1}-\Delta p H_{1} ; p H_{1}+\Delta p H_{1}\right)$, where $\Delta p H_{1}<<p H_{1}$. The first cells, inheriting from the vesicles their metabolism, added to it additional anaerobic glucose digestion and ATP spending for the synthesis of the enzymes that catalyzing the reaction of this process.

Fig. $2 c$ and Fig. $2 d$ shows the kinetics of energy metabolism of the first cells. First of them demonstrates the dependence of the rates of synthesis $V_{\text {ATФ }}^{+}$and decay $V_{\text {ATP }}^{-}$of the ATP on the concentration of ATP itself at the constant level of the cytoplasm $p H$. The second figure shows the same dependence but

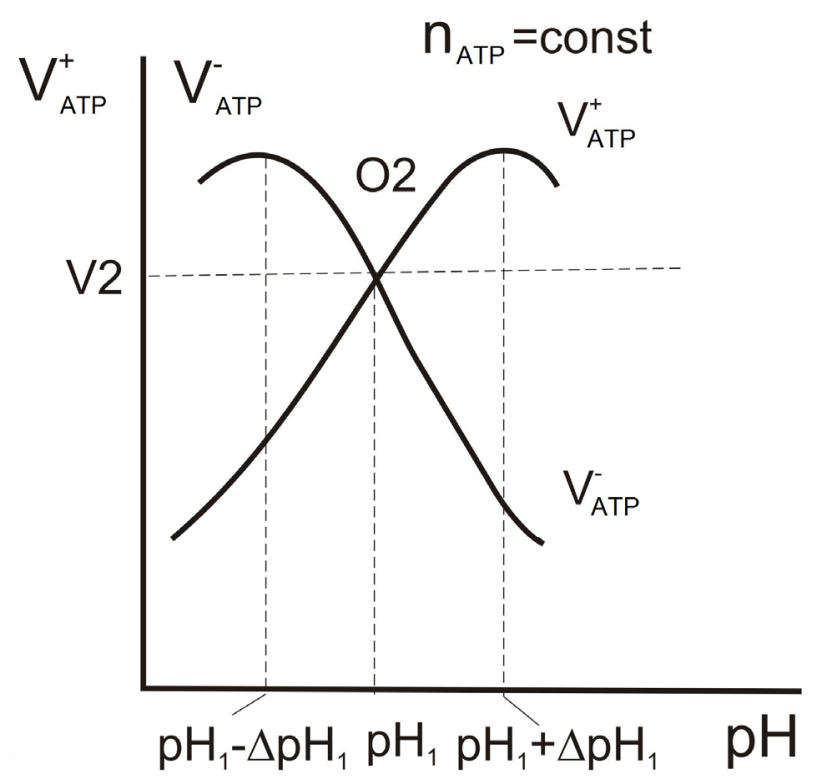

Fig. 2d. Kinetics of energy metabolism of the first cells with a constant concentration of ATP. on the $p H$ value at the constant level of the ATP concentration. Similar to metabolism of the vesicles, point $\mathrm{O}_{2}$, where is realized the balance of the rates of synthesis and decay of ATP, stable for concentration of ATP and unstable for $p H$ value. Therefore, analogy considered above case of vesicles, the oscillations of these parameters in the range $\left(n_{2}-\Delta n_{2} ; n_{2}+\Delta n_{2}\right)$ for $n_{\text {ATP }}$ and $\left(p H_{2}-\Delta p H_{2} ; p H_{2}+\Delta p H_{2}\right)$ for $p H$ took place in the first cell, but with bigger amplitudes. The balance of the rate of synthesis and decay of the main macroerg ATP implemented at the rate $V_{2}>>V_{1}$. The balance is achieved at $p H_{2}$ $<p H_{1}$, what can be explained by the magnifying of the oxidative processes, compared with the metabolism of vesicles - cells predecessor.

Fig. 3 shows the phase trajectories of the states for lipid vesicles and the first cells in the
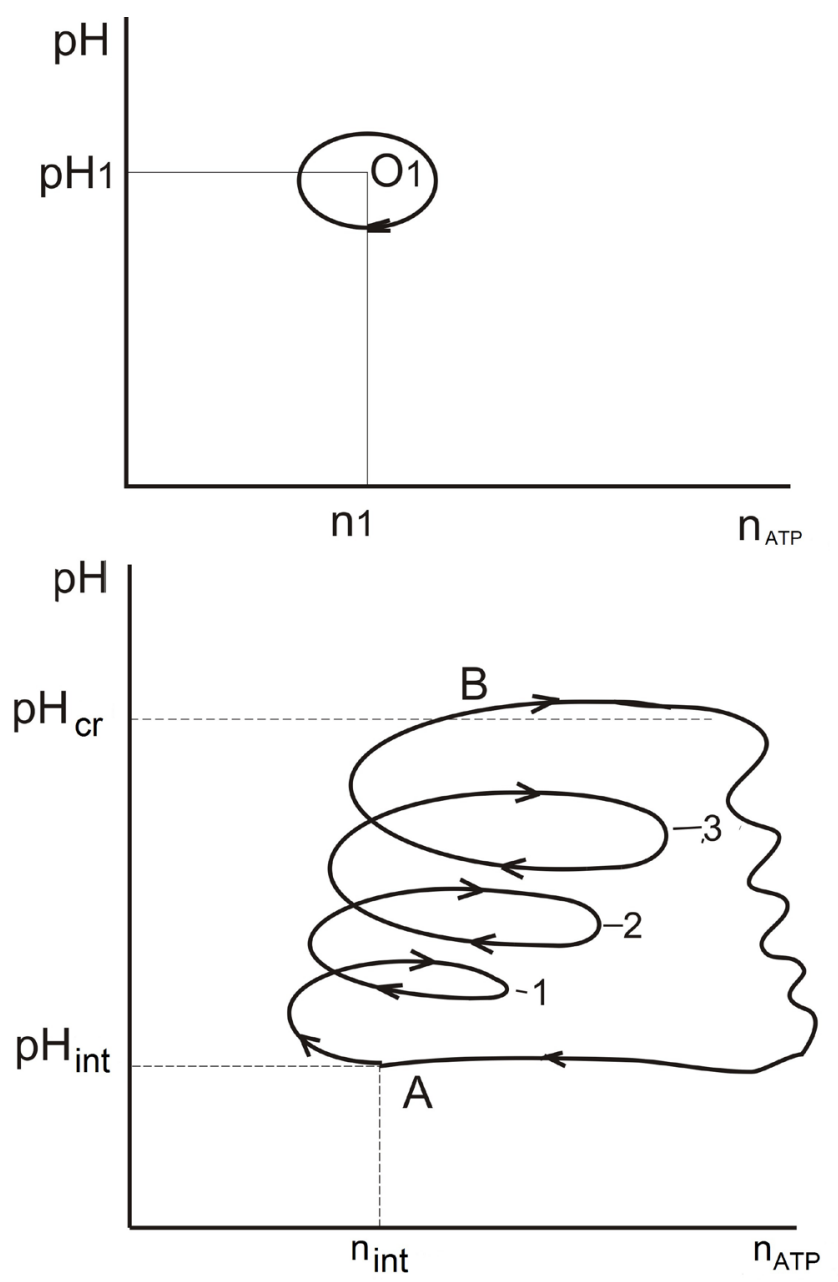

Fig. 3. Phase trajectory of the metabolism state of lipid vesicles (top) and the first cells (bottom) in the coordinates $n_{\text {ATP }}$ and $p H$. 
coordinates $-n_{\text {ATP }}$ and $p H$. On the phase plane $\left\{n_{\mathrm{AT} \Phi}, p H\right\}$, this trajectory for lipid vesicles (Fig. 3 , top) is the closed curve around the point $O_{1}$ with coordinates $\left(n_{1} ; p H_{1}\right)$ where the rates of growth and decay of the concentration of ATP and $\mathrm{H}^{+}$are strictly balanced; for the first cells (Fig. 3, bottom) this trajectory is the closed curve too, but with more complex shape. It includes clearly pronounced quasicyclic part that remains cycles of vesicles. However, $n_{\text {ATP }}$ and $p H$ values change with much more bigger growing amplitudes, shifting to the higher $p H$ values due to alkalization of the cytoplasm. It is important to note that the average values over the cycle are situated on some upward curve of their growing. As achieving the cytoplasmic neutral $p H$ values, the rate of cell alkalizing slowdown, but $p H$ value continues to grow up to maximum value despite of switching on powerful oxidative processes. After that acidity of cytoplasm monotonically increases ( $p H$ value decrease). The concentration of ATP grows monotonically too until achieve its maximum, associated with the depletion of glycogen. On this descending part of the phase trajectory one can distinguish some average line (especially at the beginning) with imposed oscillations, having much smaller amplitudes than on the ascending branch of trajectory. The terminal part of the phase trajectory caused by expenditure of ATP for enzyme pool recovery to level that was before cell division. The synthesis of the enzymes was carried out at the constant $p H$ level and at the consumed storage of glycogen, and, therefore, at the totally full inhibition of the processes of cytoplasm alkalization. On the bottom phase curve (Fig. 3) one can distinguish two functional parts: ascending one, corresponding to the mode of glycogen accumulation and descending one, corresponding to the mode of cell division. The first one we conditionally called the mode of activity, highlighting that it became the mode of activity only after the rest mode appearing in the process of evolution development.
Therefore, bottom part of the Fig. 3 shows archaic cell cycle that includes only two mentioned functional modes. Unlike archaic cells, the modern cell cycle includes also the mode (the state) of the rest. In the process of evolutionary development this state permits single-celled organisms to wait out unfavorable periods for their activity. The prerequisites for appearance of this state formed exactly during considered period of time. Let's refer to Fig. 2c. To obtain the stable point at the low ATP level, it is sufficient to increase the angle of the straight line of the decay rate $V_{\text {ATP }}$. This is achieved by growing of the concentration or activity of the group of enzymes, spending ATP on the synthetic processes (besides spending of ATP on the synthesis ATP itself) or by decreasing of the rate of enzyme decay. Then three stationary points may appear: one is discriminatory and unstable, and two others (at the lowest and the biggest ATP concentration) are stable for the ATP concentration.

\section{SELF ASSEMBLING OF THE KINETICS OF METABOLISM MODE}

The most important aspect of our analysis is the statement about automatic changing of the modes of the cell energy metabolism. We reveal that the main leading factor is changing of the $p H$ level of the cytoplasm. Exactly this factor determined the order of changing of the processes of two modes - activity and mitosis, and their mutual transitions both the way. The concentration of ATP in the cytoplasm inevitably altered too. Opposite dependence of the activity of two main groups of enzymes on the $p H$ level of the cytoplasm provided distinguishing of two energy-depending processes - synthesis of ATP and its consumption for maintaining of viability of the system [4]. The intensity of energy metabolism average over the cycle appeared to be much bigger than in the point of the rate balance of growing and decay of ATP concentration, realized in the previous evolutionary modes. 
Formation of the additional chain of the alkalizing reactions provided in the cytoplasm further progress of the general line of the living world development - intensification of the energy generation. The intensity mentioned grew up by orders of magnitude. In the cytoplasm of "precells" started automatic acidifying, including those ranges of acidity that are realized in the aqueous medium on the previous stages of the development of living world. Such changing of acidity generated all features of the viruses' interaction with predecessor of cells. For the short period of time, the microtubules, spindles and other features appeared in the cell. In this chaos and diversity only single cases of full division took place. Appearance in the cytoplasm of the inhibitors and activators of the alkalizing processes reliably provided, at first, by distinguishing in time of two modes of metabolism and, secondly, by separating of two opposing processes into distinct modes glycogen accumulation in the mode of activity and its spending in the mode of division. Reliability of lipid vesicles reproduction meant the birth of the first cells what made them immortal.

Huge, if not decisive, role in the automatically regulation of the energy metabolism plays inertness of its processes. Instant setting of the necessary mode of metabolism is impossible since the rates of this mode depend on the main parameters and for increasing or decreasing of the concentration of ATP and ions $\mathrm{H}^{+}$the cell needs time. Due to the characteristic relaxation times of these main parameters are different the hysteresis of trajectories on the phase plane $\left\{n_{\text {ATP }}, p H\right\}$ takes place when the system returns to the initial state. We showed above how cell automatically controls the characteristics of closed phase trajectory, the rates of the processes and the transition between modes of cell energy metabolism by means of additional facilities (activators and inhibitors).

\section{CONCLUSION}

In this paper we carried out the analysis of the evolutionary development of living world at the period of first animal cell formation. The conditions and possible way of transformation of lipid vesicles to the nucleated animal cells are presented. The main content of the evolutionary path was in the formation of the molecular mechanisms of the cell division (mitosis). Reliable operation of this mechanism made vesicle immortal cell. Leading physical factor defining the procedure for changing of processes and events during cell division and modes transition in their metabolism was the acidity changing of its internal environment. At the same time a necessary condition for determining the errorfree operation of these mechanisms was their reliable supply of energy.

It is important to point out the practical importance of this work. According to the statement of Teilhard de Chardin [6] about features of the evolutionary development of living systems, deconstruction of such systems goes strictly in the same way as their creation, but in reverse order. With regard to the issue of simplification of cells in carcinogenesis, this means their return to the initial stage of its evolution. Such limit simplifying corresponds to the evolutionary stages discussed in this paper. Malignant cell which turned out in the favorable conditions for its simplified metabolism in the internal environment of the organism, will wildly divide even in the absence of oxygen.

Another no less important issue than carcinogenesis is infertility of both sexes who have reached the age of their reproduction. A significant proportion of this population is healthy, but their bodies cannot reproduce gametes - haploid cells. Meiosis, the formation of gametes, is much similar to mitosis. Therefore, there are all reasons to believe that the main factor determining the order of changing of the processes and events during meiosis, as in the mitosis, is changing of the acidity of the 
cytoplasm. In our opinion, the fundamental difference between meiosis from mitosis is only in the fact that during mitosis the acidity of the cytoplasm changes automatically inside the cell, and in the case of meiosis the body is involved in this process. As maturing of gametes, they go through the ducts in the body and in the certain areas the acidity of the water surrounding environment changes providing cells with the desired mode of acidity changes in the cytoplasm of the gametes. A violation of such interaction between body and cells in meiosis can lead to infertility.

\section{REFERENCES}

1. Zaritsky AR, Pronin VS. Biofizika osnovnykh rezhimov kletochnogo metabolizma. Funktsionalnye rezhimy kletki: sostoyanie pokoya i aktivnost' [Biophysics of cellular metabolism major modes. Functional modes cells: a state of rest and activity]. Bulletin of the Lebedev Physics Institute, 2006, 12:8-18 (in Russ.).

2. Zaritsky AR, Pronin VS. Biofizika osnovnykh rezhimov kletochnogo metabolizma. Rezhim deleniya kletki (mitoz). [Biophysics of cellular metabolism major modes. Mode of cell division (mitosis)]. Bulletin of the Lebedev Physics Institute, 2006, 12:19-27 (in Russ.).

3. Zaritsky AR, Grachev VI, Vorontsov YuP, Pronin VS. Energeticheskie aspekty abiogeneza $\mathrm{V}$ atmosfere na nanokaplyakh uglevodorodnogo aerozolya [Energy Aspects of Abiogenesis in the Atmosphere on Hydrocarbon Aerosol Nanodroplets]. RENSIT, 2013, 5(2):105-125 (in Russ.).

4. Zaritsky AR, Grachev VI, Vorontsov YuP, Pronin VS. Abiogenez na etape perekhoda iz atmosfery $\mathrm{v}$ vodnuyu sredu: ot vezikul $\mathrm{k}$ protokletkam [Abiogenesis transition from the atmosphere into the hydrosphere: from vesicles to protocells]. RENSIT, 2014, 6(2):221-231 (in Russ.).
5. Lewin B, Cassimeris L, Lingappa VR, Plopper G (eds.). Cells. Moscow, Binom Publ., 2011, $951 \mathrm{p}$.

6. Teilhard de Chardin P. Le phenomene bumain. Paris, 1959. 